\title{
Surgical Oncologists and the COVID-19 Pandemic: Guiding Cancer Patients Effectively through Turbulence and Change
}

\author{
E. Shelley Hwang, MD, MPH ${ }^{1}$, Charles M. Balch, MD $^{2}$, Glen C. Balch, MD $^{3}$, Sheldon M. Feldman, MD ${ }^{4}$, \\ Mehra Golshan, MD MBA ${ }^{5}$, Stephen R. Grobmyer, $\mathbf{M D}^{6}$, Steven K. Libutti, $\mathrm{MD}^{7}$, Julie A. Margenthaler, $\mathrm{MD}^{8}$, \\ Madhu Sasidhar, MD ${ }^{6}$, Kiran K. Turaga, MD, MPH ${ }^{9}$, Sandra L. Wong, MD, MS ${ }^{10}$, Kelly M. McMasters, MD, \\ $\mathrm{PhD}^{11}$, and Kenneth K. Tanabe, $\mathrm{MD}^{12}$ \\ ${ }^{1}$ Department of Surgery, Duke University and Duke Cancer Institute, Durham, NC; ${ }^{2}$ Division of Surgical Oncology, \\ University of Texas MD Anderson Cancer Center, Houston, TX; ${ }^{3}$ Department of Surgery, Emory University, Atlanta, GA; \\ ${ }^{4}$ Department of Surgery, Montefiore Einstein Center for Cancer Care, Bronx, NY; ${ }^{5}$ Department of Surgery, Brigham and \\ Women's Hospital, Dana Farber Cancer Institute, Boston, MA; ${ }^{6}$ Oncology Institute, and Pulmonary and Critical Care \\ Medicine Institutes, Cleveland Clinic Abu Dhabi, Abu Dhabi, United Arab Emirates; ${ }^{7}$ Rutgers Cancer Institute of New \\ Jersey, New Brunswick, NJ; ${ }^{8}$ Department of Surgery, Washington University School of Medicine, St. Louis, MO; \\ ${ }^{9}$ Department of Surgery, University of Chicago Pritzker School of Medicine, Chicago, IL; ${ }^{10}$ Department of Surgery, Geisel \\ School of Medicine, Dartmouth, NH; ${ }^{11}$ Department of Surgery, University of Louisville School of Medicine, Louisville, \\ KY; ${ }^{12}$ Department of Surgery, Massachusetts General Hospital, Boston, MA
}

\begin{abstract}
Background. The COVID-19 pandemic has posed extraordinary demands from patients, providers, and health care systems. Despite this, surgical oncologists must maintain focus on providing high-quality, empathetic care for the almost 2 million patients nationally who will be diagnosed with operable cancer this year. The focus of hospitals is transitioning from initial COVID-19 preparedness activities to a more sustained approach to cancer care.
\end{abstract}

Methods. Editorial Board members provided observations of the implications of the pandemic on providing care to surgical oncology patients.

Results. Strategies are presented that have allowed institutions to successfully prepare for cancer care during COVID-19, as well as other strategies that will help hospitals and surgical oncologists manage anticipated challenges in the near term. Perspectives are provided on: (1) maintaining a safe environment for surgical oncology

(C) Society of Surgical Oncology 2020

First Received: 17 May 2020;

Published Online: 14 June 2020

K. K. Tanabe, MD

e-mail: ktanabe@partners.org care; (2) redirecting the multidisciplinary model to guide surgical decisions; (3) harnessing telemedicine to accommodate requisite physical distancing; (4) understanding interactions between SARS CoV-2 and cancer therapy; (5) considering the ethical impact of professional guidelines for surgery prioritization; and (6) advocating for our patients who require oncologic surgery in the midst of the COVID-19 pandemic.

Conclusions. Until an effective vaccine becomes available for widespread use, it is imperative that surgical oncologists remain focused on providing optimal care for our cancer patients while managing the demands that the COVID-19 pandemic will continue to impose on all of us.

The COVID-19 pandemic has required that health systems and medical care providers critically assess existing treatment algorithms across all healthcare settings. Cancer treatment is complex, with a range of urgencies for intervention, a large variety of potential treatment approaches, and numerous treatment sequencing options. Moreover, cancer patients are often elderly and have comorbidities, and cancer therapies frequently employ immunosuppressive agents. These factors converge to further intensify the complexities of cancer treatment during the COVID-19 pandemic. It is important to be mindful that, even with the 
devastating consequences of the COVID-19 pandemic, over 1.8 million Americans will be diagnosed with cancer this year, and over 600,000 will die of their disease. ${ }^{1}$ Thus, until an effective vaccine becomes available for widespread use, it is imperative that surgical oncologists remain focused on providing optimal care for our cancer patients, while managing the demands that the COVID-19 pandemic will continue to impose on our health system and our providers.

To this end, a panel of editors of the Annals of Surgical Oncology were invited to reflect upon those strategies that have allowed institutions to successfully prepare for cancer care during COVID-19 as well as others that will help hospitals and surgical oncologists manage the expected challenges at the intersection of COVID-19 and cancer care. A summary of key measures that have established a framework from which to address these issues is provided, as well as additional strategies for managing resumption of surgical care in cancer patients. The panel provides perspectives on: (1) creating a safe environment for surgical oncology care, (2) redirecting the multidisciplinary model to guide surgical decisions, (3) harnessing telemedicine to accommodate requisite physical distancing, (4) understanding interactions between SARS CoV-2 and cancer therapy, (5) considering the ethical impact of professional guidelines for surgery prioritization, and (6) advocating for our patients who require oncologic surgery in the midst of the COVID-19 pandemic.

\section{DEVELOPING AN INFRASTRUCTURE TO MEET THE DEMANDS OF CANCER CARE AND COVID-19}

\section{Establishing a COVID Taskforce}

The initial action of most medical centers' response to the COVID-19 pandemic was to create a multidisciplinary taskforce ("incident command center") including executive leadership, infectious disease experts, and department leaders to provide oversight, create policy based on available scientific evidence, and allocate resources for managing the crisis. Further, the taskforce is typically responsible for communicating a unified message regarding the evolving pandemic across the organization, which is critical for caregiver wellbeing and safety. Utilizing a realtime dashboard developed for the purposes of managing the approach to the COVID-19 pandemic, the taskforce sets organizational priorities in a rapidly changing environment. This has resulted in tiers of care for our oncologic patients, providing a framework for which patients should be treated with standard treatment pathways during the COVID-19 pandemic versus those who should be considered for alternative pathways of care.
One critical role of the taskforce has been to engage with other regional leaders to share best practices in a rapidly changing environment. For example, a weekly New Jersey-wide cancer program teleconference was organized, with participation from many of the cancer programs across the state representing all the largest healthcare systems. The teleconference afforded all the programs the opportunity to share common experiences and best practices as well as set treatment standards informed by national guidelines but tailored to some of the unique challenges in the state. As another example, hospitals across Boston have coordinated a regular meeting to exchange utilization and capacity data as a strategy of balancing load and minimizing the likelihood of implementing crisis standards of care asymmetrically across hospitals. While such strategies were considered advisable in the past, they are now an essential part of regional strategy for healthcare delivery.

\section{Creating a Safe Hospital and Clinic Environment}

A fundamental focus on safety in the hospital and the clinic was an early and essential step for minimizing risk of exposure to all patients and caregivers, especially for those who needed oncologic care. The pandemic has forced most institutions to limit activity at the hospital and clinics to the most essential tasks to reduce potential viral transmission and enhance efforts to achieve social distancing for patients and staff. In some countries, including Italy, there have been coordinated efforts to segregate either entire hospitals or hospital units for COVID-19 patients, allowing physical separation of patients with greatest transmission risk from those who are most vulnerable. Special training in biosafety measures has been required, and protocols have been put in place in every hospital, clinic, and cancer center to optimize the safety of caregivers in these areas. ${ }^{2}$ Distancing requirements have led to an increased need for electronic communication devices such as smartphones and laptops, which have been provided to patients with COVID-19 infection to enhance remote communication between patients and caregivers and family members while limiting exposure. Enhanced cleaning protocols should be implemented in these areas and other "hot spot" areas including the Emergency Department and Occupational Health. Protocols and dedicated teams have been created for safe patient transport and response to acutely deteriorating patients.

The pandemic has required that the number of access points to the hospital and clinics be significantly reduced, and if available, thermal cameras have been used to assess all people entering - patients, visitors, and caregivers-for potential COVID-19 infection. In many hospitals, no visitors are allowed; in others, the number of visitors allowed 
has been significantly restricted. Prior to in-person visits at hospital clinics, patients are now contacted by telephone and screened for symptoms of potential COVID exposure or infection, with those reporting possible symptoms being directed to testing areas and then triaged accordingly. Among cancer patients, the European Society of Medical Oncology (ESMO) guidelines recommend testing for COVID infection prior to chemotherapy infusions, ${ }^{3}$ although it is acknowledged that shortage of tests preclude this strategy at many hospitals. Employee travel has been restricted in most institutions to reduce exposure of caregivers to COVID-19 in high-risk areas; And at a minimum, employees are required to disclose travel upon return so that appropriate testing or quarantine may be implemented.

\section{Protecting the Healthcare Workforce}

In response to the COVID-19 pandemic, organizations have enacted plans centered around "caring for patients" and "caring for caregivers" core values of healthcare organizations around the world. The downstream effect of protecting healthcare workers who care for patients with cancer is a reduction in potentially life-threatening COVID-19 infections in our immunosuppressed cancer patients. New tools and resources have been required to support unfamiliar models of work for caregivers. Reassignment of clinicians has been required to meet the evolving demands of the pandemic, in particular at ambulatory respiratory care clinics, COVID-19 inpatient floors, and ICUs. Rapid retraining of caregivers to fill critical positions which are outside of their traditional scope of practice have been accomplished via web-based platforms. Live intranet-based courses have been developed covering topics which include ICU workflow, update of COVID-19 treatment protocols, management of sepsis and acute respiratory distress syndrome (ARDS), and ventilator management. Some states have provided healthcare workers with civil liability protection, recognizing the need for many to work outside their area of specialty.

Creative work hour scheduling is one effort that attempts to minimize exposure among healthcare team members. For example, some hospitals have created teams of physicians within a specialty who rotate coverage for hospital and clinic patients ( $\mathrm{A}$ and $\mathrm{B}$ teams). Team A provides in-person care in the hospital and clinic, while team $\mathrm{B}$ provides care using digital platforms from remote locations. Team A and B switch roles on a weekly basis. Such scheduling strategies are intended to limit the spread of infection at the workplace within a group of specialists and staff. The mean asymptomatic period during incubation time is 5.1 days; and following high-risk exposures and a 7-day monitoring period, the estimate for missed cases is 21.2 per $10,000,{ }^{4}$ thereby providing theoretical support for a weekly rotation schedule. Simultaneous cross-coverage of infusion, clinic, and in-hospital patients by medical oncologists is another strategy to reduce the size of the required in-hospital physician workforce to reduce their potential exposures. This is feasible given the overall reduction in patients at the hospital due to triage. A large percentage of those providing indirect patient care (secretarial support and administrative services) are generally enabled to work from home via secure digital platforms. This too requires rapid training of these staff and, in many instances, provision of hardware.

Access of caregivers to protective equipment (PPE) has clearly emerged as essential for maintaining a safe workforce. 5 PPE use education has been one of the most challenging aspects of managing communications. Over the course of the pandemic, recommendations from the WHO and CDC have changed and have often not taken into account local shortages of certain types of PPE. To rapidly disseminate correct use of PPE and assessment of patients with respiratory symptoms, electronic communication through the COVID-19 intranet site has been utilized.

Preservation of PPE while simultaneously providing a safe environment for caregivers has been critically important. Protocols have been established and shared with caregivers via the COVID-19 intranet site for extended use and limited reuse of N95 masks and eyewear for those in high-risk environments. In addition, modified donning and doffing protocols for safe recycling are shared via the COVID-19 intranet site. In many centers, caregivers have been provided a storage bag for repeated N95 use during the day. At the end of a shift, the storage bags are discarded, and N95 masks are placed in designated collection bags for resterilization. ${ }^{6}$ Large-scale reprocessing of masks using hydrogen peroxide vapor has been approved by the US Food and Drug Administration and is currently being utilized to establish a backup stock of PPE for emergency use in some areas.

Reduction of in-person meetings is in alignment with the goal of physical distancing. Meetings within healthcare organizations have uniformly been transitioned to virtual meetings using secure digital platforms. For caregivers with direct patient contact and without a way to self-isolate at home, on-site or hotel rooming may be provided to limit exposure of a caregivers' family members during the pandemic. $^{7}$

\section{Modifying Operating Room Workflows and Case Reduction}

Elective operative procedures for cancer patients have been cancelled in most organizations to prioritize resources 
for the care of patients with COVID-19. Many guidelines for resource allocation have been proposed, including one from the American College of Surgeons, ${ }^{8}$ and they are covered in more detail below. For patients undergoing planned essential cancer operations, COVID testing should ideally be performed within $72 \mathrm{~h}$ of the planned procedure; however, few hospitals have had access to sufficient numbers of tests to incorporate this into their testing algorithm. For asymptomatic patients that test negative for COVID-19, anesthetic and surgical procedures are conducted under standard operating protocols. For patients who test positive for COVID-19, surgical procedures are delayed when possible, both to minimize exposure to healthcare workers and to reduce postsurgical risk. For those patients who test positive for COVID-19, whose symptoms are consistent with COVID-19 infection, or in whom testing is not available or possible (e.g., emergent cases), enhanced OR management protocols ${ }^{9}$ must be in place to reduce viral exposure to anesthesia, surgical, and nursing teams (Table 1).

\section{Supporting the Wellbeing of Healthcare Teams}

Significant levels of psychological stress, including depression, anxiety, and insomnia, have been documented among healthcare workers involved in the care of patients with COVID-19. ${ }^{10}$ To proactively address this, hospitals have looked to provide resources, including professional coaching, courses focused on wellbeing and resilience, and stress management, through virtual support groups. Virtual exercise classes are also being offered to assist caregivers with maintaining physical fitness during the period of physical distancing.
Closures of schools and daycare centers have created an additional barrier for healthcare employees to report to work away from home. Hospitals and medical centers do not operate daycare centers large enough to accommodate children for all their employees. Moreover, even an attempt to scale up on-site facilities runs at odds with institutional goals of social distancing. For many employees, the ability to work from home and simultaneously provide childcare serves as a workable solution. For others, hospitals have contracted with vetted reputable outside organizations to provide childcare support at reduced rates for their employees.

Health systems have taken decisive measures to ensure the safety and wellness of their most important resources: their staff and patients. The actions that the pandemic has required of all healthcare systems have helped to address some long-standing issues and may allow hospitals to emerge from this challenge as stronger and more effective institutions.

\section{MULTIDISCIPLINARY CANCER TEAMS}

The multidisciplinary approach is a familiar cornerstone of cancer treatment, and repurposing this framework during the COVID-19 pandemic allows care teams to continue to provide patient-centered cancer care with excellent outcomes, while effectively pursuing institutional priorities such as patient and staff safety, social distancing, and conservation of resources including PPEs. ${ }^{11}$ In response to the COVID-19 pandemic, many cancer centers have expanded the multidisciplinary team model to help manage oncologic care (Table 2). Many guidelines for surgical oncology triage have been advanced during the COVID-19 pandemic by professional societies and hospitals., ${ }^{3,12-16}$

TABLE 1 Operating room management to protect caregivers during procedures on COVID-positive patients ${ }^{9}$

\begin{tabular}{|c|c|c|}
\hline Prior to operation & During intubation and operation & Following operation \\
\hline $\begin{array}{l}\text { Do not bring patient to holding area to limit exposure } \\
\text { to other patients and caregivers }\end{array}$ & $\begin{array}{l}\text { Double glove and remove outer } \\
\text { gloves after intubation }\end{array}$ & $\begin{array}{l}\text { Recover patient in airborne infection isolation } \\
\text { room }\end{array}$ \\
\hline $\begin{array}{l}\text { Place signs on OR door to limit unnecessary caregiver } \\
\text { exposure in OR }\end{array}$ & $\begin{array}{l}\text { Intubation performed by most } \\
\text { experienced team member }\end{array}$ & $\begin{array}{l}\text { Allow time between cases for adequate } \\
\text { clearance of airborne infectious } \\
\text { contamination }^{\mathrm{a}}\end{array}$ \\
\hline Place HEPA filer on anesthesia circuit & $\begin{array}{l}\text { Avoid awake fiber-optic intubation } \\
\text { when possible }\end{array}$ & $\begin{array}{l}\text { Clean and disinfect room and equipment using } \\
\text { approved disinfectants }\end{array}$ \\
\hline \multirow{3}{*}{$\begin{array}{l}\text { Caregivers should wear PPE and follow airborne and } \\
\text { contact precautions, including eye protection }\end{array}$} & Consider rapid sequence intubation & \\
\hline & $\begin{array}{l}\text { Use disposable plastic covers on } \\
\text { equipment to reduce } \\
\text { contamination }\end{array}$ & \\
\hline & $\begin{array}{l}\text { Nonventilated patients should wear a } \\
\text { mask }\end{array}$ & \\
\hline
\end{tabular}

\footnotetext{
${ }^{a}$ Between 8 and 207 min to achieve removal efficiency of $99.9 \%$ depending on number of air exchanges per hour in OR
} 
TABLE 2 Goals to guide COVID-19 response for surgical oncology planning

\begin{tabular}{ll}
\hline COVID-19 planning priorities & COVID-19 emergence preparedness \\
\hline $\begin{array}{l}\text { Assure optimal long-term clinical outcomes for } \\
\text { patients with cancer }\end{array}$ & $\begin{array}{c}\text { Prospectively collect patient information on patients with surgical delay, including } \\
\text { patients undergoing neoadjuvant therapy }\end{array}$ \\
$\begin{array}{l}\text { Minimize the risk of infection or exposure among } \\
\text { patients and staff }\end{array}$ & $\begin{array}{r}\text { Work with multi-D team and other disease site teams toassign priority designation for } \\
\text { every patient experiencing surgical delay }\end{array}$ \\
$\begin{array}{l}\text { Protect patients from treatment-related side effects, } \\
\text { such as immunosuppression }\end{array}$ & $\begin{array}{c}\text { Correlate surgical resource needs (OR time, inpatient beds, ICU beds) with institutional } \\
\text { availability to plan resumption of cancer surgery schedule }\end{array}$ \\
$\begin{array}{l}\text { Preserve vital resources within the healthcare } \\
\text { system }\end{array}$ & $\begin{array}{c}\text { Prioritize ongoing communication with patient, multi-D team } \\
\end{array}$ \\
\hline
\end{tabular}

However, guidelines appropriate for one hospital or region may not be appropriate for another, given that hospitals and regions are in different phases of the pandemic. In addition, the appropriateness of some guidelines shifts as the impact from COVID-19 changes over time. It is thus appropriate and essential to leverage multidisciplinary teams to develop care pathways, provide recommendations for individual patients, triage when necessary, and redesign workflows. Some small studies have reported dual concerns for cancer patients of both increased risk of contracting the viral illness and higher rates of surgical morbidity and mortality among affected patients. ${ }^{17,18}$ While evidence is still limited, such studies underscore the need to balance the numerous competing concerns including patient safety, health system resources, everchanging hospital mitigation strategies, optimal cancer treatment, and consideration of patient comorbidities. ${ }^{19}$ Bartlett et al. have provided an early overview of key surgical considerations that must be taken into account broadly for all cancers as well as for specific cancer types. $^{13}$

Multidisciplinary discussion is collaborative and incorporates the specific risks and benefits from the perspectives of different specialties. Surgeons will consider risks of exposure to COVID-19 from an operation, adequacy of blood bank supplies, potential need for a precious ICU bed postoperatively, and risks to staff including nurses and anesthesiologists. Radiation oncologists will consider the risks to patient and staff and challenges faced by some patients undergoing radiation therapy in a setting in which visitors are not allowed. Medical oncologists will consider avoidance of immunosuppressive treatments and use of regimens that can be administered at home. All team members will consider treatment efficacy, impacts of potential treatment delays, risks of travel, the number of required trips to the medical center, and challenges of clinical trial participation. As multidisciplinary teams converge to balance these competing priorities, several key areas merit careful consideration (Fig. 1).

\section{Patient Assessment and Treatment Planning}

In medicine, every action has inherent risks and benefits, and this balance may be shifted during the COVID-19 pandemic. One must consider whether the recommended cancer care and potential exposure to COVID-19 places the patient at higher risk than delaying care in favor of quarantine until the pandemic has eased. In some patients, delay of their cancer treatment may be in their best interest. Patients that harbor the novel coronavirus may have poor outcomes during treatment, in particular if rendered immunocompromised secondary to cancer therapy. As a result, the multidisciplinary team must consider nonstandard approaches to treatment. The COVID-19 pandemic has forced flexibility and thinking outside the box when considering treatment options for each patient. Algorithms in cancer care are vital to consistent outcomes, but strict adherence to treatment algorithms cannot singularly override consideration of many COVID-19-related priorities.

Patients will be naturally anxious about the suggestion of a delay in their cancer treatment. They may be provided with reassurance that a multidisciplinary team has determined that the planned delay will not impair overall outcome and that the risk of a poor outcome from COVID19 exposure is greater than the risk of treatment delay. It is important to review with the patient the treatment plan and rationale for the recommendations as well as schedule regular updates as resources and capacity change within the health system. A new diagnosis of cancer confers a level of psychological stress on every patient, and the COVID-19 pandemic poses additional emotional stress. Thus, multidisciplinary teams should also be prepared to recognize patients who are struggling to manage their mental health and provide interventions for relief.

\section{Ambulatory Patient Triage}

Defining urgency of ambulatory care in a multidisciplinary manner may allow some patients to have their appointments safely delayed. Each cancer disease program 


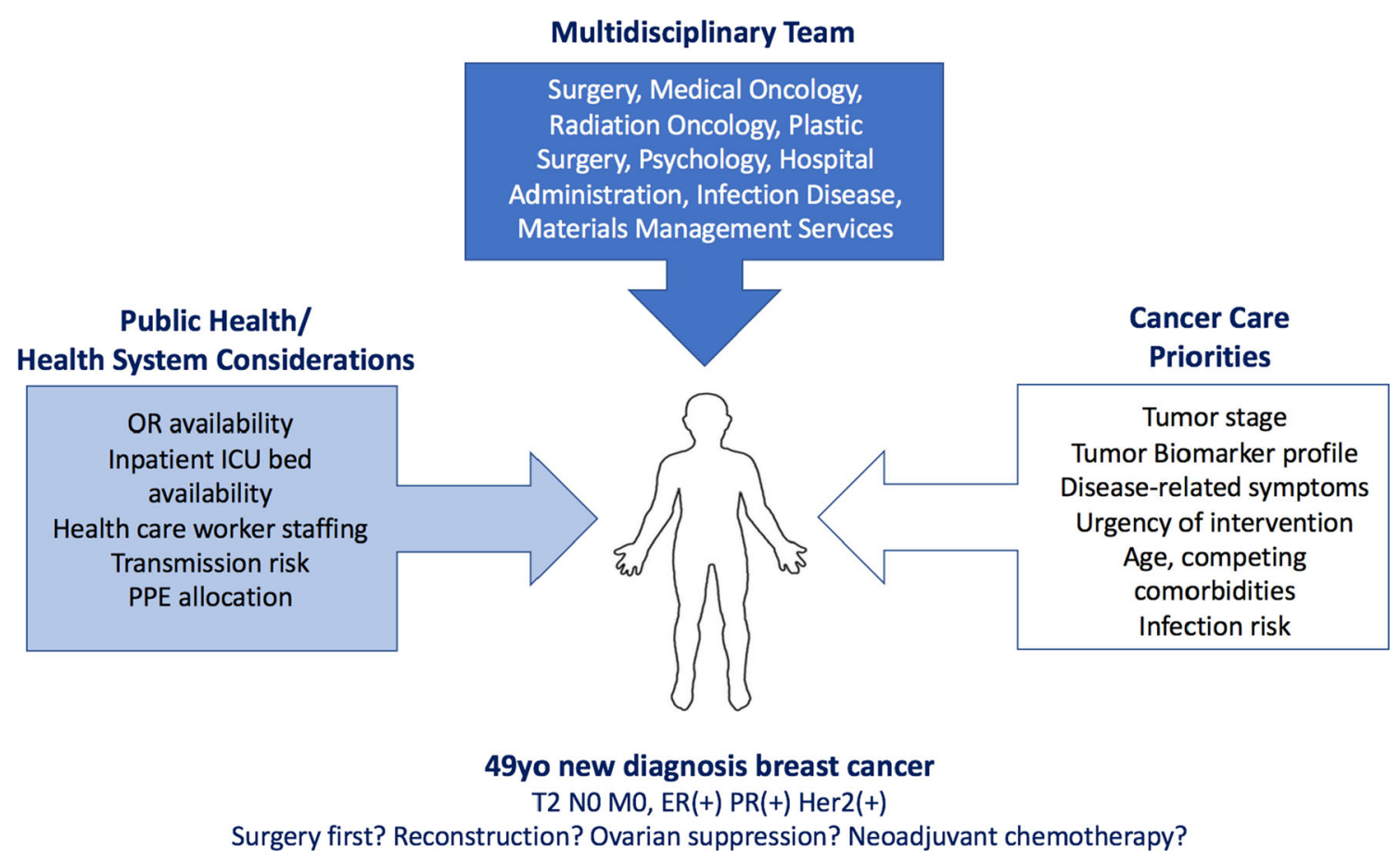

FIG. 1 Complex cancer care and public health priorities in management of operable cancer. The example demonstrates the myriad issues that converge on a patient with a new diagnosis of

should develop priority categories based on individual demographics, imaging and pathology reports, and tumor phenotype. Teams must determine the order in which patients should be seen and by which team members. In addition, treatment teams may determine which patients can be effectively managed using a telehealth platform. For example, one strategy involves new patients with operable cancers being seen in person by a surgeon, and a medical oncologist providing consultation remotely, minimizing the number of direct encounters with the patient. In each case, the patient and their family are reassured that they will receive safe, effective, and time-sensitive care in this changing environment. Multidisciplinary meetings to establish treatment recommendations for each patient should be accompanied by clear documentation of the treatment recommendations and the disciplines present during the discussion. Published society guidelines should be used as resources whenever possible to guide management recommendations.

\section{Prioritization of Cancer Surgeries After the COVID-19 Surge}

Emergence following the COVID-19 surge requires the same intensity of planning and resourcefulness that was initially required to address this crisis. Planning for resumption of surgical cancer care requires the breast cancer, increasing the complexity of cancer care decisions. Comparable considerations apply to other operable tumors

development of patient priority categories based on urgency of intervention, postoperative resource requirements, and projected availability of these resources across the institution. In Italy, one of the first countries to suffer from devastation and resource constraints from the pandemic, a simple four-tier prioritization schema incorporating urgency, tumor factors, and patient factors was instituted successfully, providing an effective and transparent process. ${ }^{20}$ Establishing a priority list of patients with delayed operations requires communication not only within a cancer disease group but also across disease groups to truly optimize resource utilization. The multidisciplinary approach is again emphasized, recognizing the importance of integrating additional information such as nurse staffing levels, blood bank supplies, and ICU beds. There are active efforts by both the National Cancer Institute (NCI) and international groups to examine the impact of surgery in both COVID-19-confirmed patients and patients with cancer affected by delay of surgery or radiation that will yield important insights into outcomes in these affected cohorts (e.g., the UK National Institute for Health Research Global Health Research Institute NCT04323644). These studies will provide additional evidence on the effect of surgical delay and nonstandard management of cancer on cancer outcomes. 


\section{Patient Communication}

There has never been a time when patient communication has been more vital as during the present COVID-19 pandemic. Conversations with patients, in particular about treatment delay, are difficult but essential, especially as the long-term consequences of treatment delays are often not well established. A multidisciplinary approach allows for more uniform and consistent messages to patients. Conveying consensus among team members to a patient helps alleviate their anxieties, in particular when treatment recommendations do not adhere to standard established guidelines.

The use of a multidisciplinary approach provides an organized rational framework to allow mitigation of the impact of COVID-19 without compromising the excellent outcomes that have been achieved in cancer care. ${ }^{11}$ Moreover, it can be an effective tool to manage patient safety while reallocating resources.

\section{PANDEMIC-DRIVEN ACCELERATION OF TELEMEDICINE IN SURGICAL ONCOLOGY}

Albert Einstein famously said: "In the midst of every crisis, lies great opportunity." It may be more pragmatic to consider that a crisis accelerates inevitable change and that the opportunity presented in the midst of the COVID-19 pandemic lies in examining burgeoning medical technology. "Telemedicine" is an encompassing term which refers to the exchange of medical information through electronic communications. These technologies have gained widespread attention, but adoption has not been universal, noting significant barriers associated with the expenses of implementation, reimbursement challenges, and specific services that require an in-person visit for a physical examination or imaging and labs. For many patients, limitations are based on a lack of access to a computer/mobile device as well as lack of broadband access in more rural areas. In 2016, only $15.4 \%$ of physicians worked in practices that used telemedicine, and rates for surgeons were even lower $(11.4 \%){ }^{21}$ However, with the COVID-19 pandemic, interest in and use of telemedicine has accelerated to slow community spread of the virus and limit exposure of other patients and staff members while providing essential non-COVID healthcare.

\section{The Role of Telemedicine Prior to COVID-19}

Even before the pandemic, telemedicine was being evaluated for much-needed oncology services secondary to national shortages of cancer specialists and limited access to subspecialty care for patients in rural or underserved areas, including a patient's ability to obtain second opinions or a provider's ability to discuss cases at a multidisciplinary tumor board meeting. Efforts such as the Extension for Community Healthcare Outcomes (ECHO) project, ${ }^{22}$ an evidence-based method that links interdisciplinary specialists with community-based practitioners using web conferencing technology, were being implemented across specialties to enable better management of complex conditions locally, including a focus on cancer care. For surgeons, uptake of telemedicine was being considered in the preoperative and postoperative phases of care.

\section{Potential Roles for Telemedicine}

Many studies found that, for selected conditions, preoperative diagnosis via telemedicine was as accurate as interventions carried out in conventional clinics. ${ }^{23}$ Better incorporation of "store-and-forward" (Table 3) for imaging studies, pathology reports, and other medical records will improve the efficiency of real-time telemedicine visits and add to patient satisfaction. In many ways, this mirrors the steps needed for specialty consultation appointments, since these components of a patient's file are collected and forwarded for review in advance of seeing the patient. Substituting a virtual visit for an in-person meeting with a surgeon in advance of a scheduled operation has yet to be implemented broadly and would need to account for issues around OR scheduling to allow first-time in-person meetings in the preoperative area and then questions around tenets of the informed consent process. Indeed, the role of telemedicine for surgeons is not well studied, and the extent of implementation is unknown but has most certainly been accelerated by the pandemic. Other implications of preoperative telemedicine visits have yet to be evaluated robustly, taking into account issues of data security and patient privacy as well as broader implications for the patient-doctor relationship, including the patient perspective on the benefits (and downsides) of a virtual preoperative visit.

Postoperative assessments conducted by telemedicine, mainly for drain care or wound assessments, could decrease the burden of ambulatory clinic visits. Further, a handful of studies showed that patient satisfaction with virtual visits compared favorably with usual care, noting a reduction in "unnecessary" trips to see their doctors, with attendant savings in costs associated with travel and time away from work. ${ }^{23}$ For postoperative care, many surgeons were already using telephonic check-ins or communication via patient portals embedded in electronic medical records as postoperative hospital stays were becoming shorter. Common uses are already in place for discussion of pathology results and postoperative symptom management. 
TABLE 3 Commonly used telemedicine terms

\begin{tabular}{ll}
\hline Term & Definition \\
\hline Store-and-forward & $\begin{array}{c}\text { Describes services that are not real-time. Broadly speaking, "store-and-forward" describes the exchange of data } \\
\text { (including imaging, photos, and other patient records) that are transmitted asynchronously with a live patient encounter } \\
\text { Commonly used "live video" or videoconferencing describes a real-time (or synchronous) video and audio patient } \\
\text { encounter }\end{array}$ \\
Live video & $\begin{array}{l}\text { Refers to the location of the patient receiving telemedicine services. In many cases, originating site were previously } \\
\text { limited to "established medical sites" }\end{array}$ \\
Originating site & $\begin{array}{l}\text { Refers to a patient location ("originating site") staffed by an on-duty medical professional who acted as a local } \\
\text { facilitator. Established medical sites included clinics, hospitals, or certain other types of medical facilities with a local } \\
\text { sites } \\
\text { facilitator who helps transmit information using digital assessment or monitoring devices as part of a telemedicine visit } \\
\text { Refers to the location of the licensed provider who is rendering telemedicine services. Where applicable, facility fees } \\
\text { may be covered and reimbursed in addition to professional fees for the service provided }\end{array}$ \\
\hline
\end{tabular}

Beyond the perioperative phases of care, there is tremendous opportunity in evaluating a role for telemedicine in other aspects of oncology care. One major area that many experienced as a relatively seamless transition during COVID-19 pandemic was videoconferencing platforms for team conferences and tumor boards. For hospitals facing resource constraints (e.g., PPE), which precluded all but highly selected elective surgical cases, the need and desire for consensus-driven decision-making was more apparent than ever, especially given considerations for triage and alternate treatment strategies. Indeed, some have noted greater conference participation as virtual platforms were made available, heralding a welcome change for many.

\section{The Impact of COVID-19 on Telemedicine}

During the pandemic, many ambulatory clinic visits for interval oncologic surveillance were converted to telemedicine visits rather than being postponed. However, for many of these "routine" follow-ups, concomitant needs for imaging and labs precluded wholesale transition of these appointments to a virtual platform since a visit to a medical facility was still required for tests which serve as an adjunct to physical examination. As patients were heeding to social distancing and sheltering in place, research staff were reassigned to work remoting to the extent possible, putting accrual for clinical trials and nonessential in-person research visits on hold unless a virtual format was able to be developed and implemented \{reference ASO Pawlik et al. COVID article in press $\}$. Indeed, the FDA issued guidance recommending "alternative methods for assessments, like phone contacts or virtual visits". 24

Under the 1135 waiver authority and Coronavirus Preparedness and Response Supplemental Appropriations Act, the Centers for Medicare and Medicaid Services (CMS) expanded the scope of and payments for telemedicine service on a temporary and emergency basis, effective March 6, 2020. ${ }^{25}$ Prior to this, Medicare only covered limited telemedicine visits and placed restrictions on qualified beneficiaries (patients) and originating site, such that the patient had to live in a designated rural area and had to receive the service at an established medical site (Table 3). To specifically allow for home-based telemedicine, many prior requirements were eliminated. Importantly, reimbursement parity for telemedicine visits was addressed, noting that Medicare will make payments at the same rate as regular in-person visits. For other types of services such as virtual check-ins or e-visits, patients must have an established relationship with the physician and follow established time intervals following or preceding another evaluation and management (E\&M) service or procedure. These services include brief communications and can include an array of communication technology modalities, such as telephone or use of online patient portals.

\section{Expanding Opportunity and Access to Telemedicine}

With the need to quickly move to provide care while minimizing risks during the COVID-19 pandemic, about half of state medical boards issued temporary waivers or allowances for in-state licensure requirements related to telemedicine (since the usual requirement is that physicians engaging in telemedicine are licensed in the state in which the patient is located). ${ }^{26}$ In addition, many states changed regulations around patient consent and Medicaid billing to rapidly enable the provision of care via telemedicine. This has led to rapid uptake of virtual visits using various insurance carrier-based platforms as well as stand-alone (third-party vendor) products. Waivers have been variable in terms of coverage determinations for audiovisual versus audio-only (e.g., telephonic) visits and synchronous versus asynchronous services (Table 3 ). In some states, services 
that would normally require an in-person visit such as writing prescriptions, including those for controlled substances, were waived as well.

As with most change efforts, due consideration should be given to how to achieve "sticky change" in postCOVID times. Interestingly, the future of telemedicine seems assured since the accelerated advances being made (and which continue to be made) during the pandemic are unlikely to dissipate and appear to have been widely embraced. Of course, there will need to be an overarching assessment of alignment between health systems' strategic goals and continued promulgation of telemedicine. Ease of use, quality of care, and satisfaction with virtual encounters will need to be assessed on both the provider and patient side. Physicians may appreciate increased flexibility with visits, possibly allowing for more visits in a given day and removing inefficiencies that are created by last-minute cancellations and over-crowded clinics. However, real work will need to be done to assess barriers and facilitators so that telemedicine services are as effective and efficient as possible. From a workflow perspective, early lessons (and indeed, lessons on the frontline) include rapid ascertainment of technology skills, not only to be facile with operating the selected platform but also to allow for patient engagement and rapport while managing screens and the electronic medical record. Patients who had a preappointment orientation appear to be better prepared for telemedicine encounters, which allowed for more focus on the appointment and not the associated technology.

\section{Telemedicine Beyond the Pandemic}

Issues which are likely to require immediate attention at a national level are evaluation of the emergent/temporary CMS policies including state-level licensure and regulatory decisions put into place during the pandemic. Payment rates for telemedicine services may need to be revised since the current list of covered services is limited. If homebased visits continue, issues around reimbursement of facility fees, for both the originating and distant site (Table 3), will need to be evaluated to ensure that costs around supporting the technology infrastructure are covered and that there is fair reimbursement for the services provided. Expansion of telemedicine services may create opportunities for savings under episode-based bundled payments. Technology barriers which had previously been raised will resurface, including issues with bandwidth for rural residents and functional technology platforms for data collection and secure, Health Insurance Portability and Accountability Act (HIPAA)-compliant communications. Forward thinking about healthcare disparities is also warranted, understanding that lack of access to technology may exacerbate inequities.

\section{INTERACTIONS BETWEEN SARS-COV-2 AND CANCER THERAPY}

The experience with COVID-19 in Europe and Asia has established that a cancer diagnosis places a patient at much higher risk for morbidity and mortality and perhaps at higher risk for SARS-CoV-2 infection. ${ }^{27-29}$ For example, patients with lymphoma, leukemia, and lung cancer on active therapy can suffer mortality as high as $50 \%$. It is critical, therefore, to minimize the risk of SARS-CoV-2 exposure for patients with a cancer diagnosis, especially those receiving active cancer treatment. Absent a vaccine or medications that can block viral entry, the only tools available at the moment are physical separation and PPE.

\section{COVID-19 and Immunotherapy}

The use of immunotherapy for the treatment of cancer is now considered mainstream. The number of patients with common epithelial cancers treated with immune checkpoint inhibitors (ICI) has been increasing, especially for patients with tumors of the lung, head and neck, kidney, and melanoma. With respect to the administration of checkpoint inhibitors (anti-CTLA-4 and anti-PD-1/PD-L1), there are essentially two major concerns that have been described. $^{30}$

First, pulmonary toxicities from ICI can either confound or exacerbate manifestations of COVID-19. Lung toxicity from ICI can be life threatening. The incidence of ICIrelated pneumonitis can be as high as $10 \%$ with combination therapy. ${ }^{30}$ While this is not the most common adverse event seen with ICI therapy, it is the most lethal with a mortality approaching $34 \%$. Since patients with COVID-19 can manifest an immune pneumonitis, it can be a challenge to discern whether such patterns on chest X-ray (CXR) or computed tomography (CT) scans are due to ICIinduced toxicity or a manifestation of COVID-19. It is also not known whether the combined morbidity of ICI-induced pneumonitis and COVID-19-related lung injury are additive or synergistic with respect to mortality. This is particularly important since autoimmune pneumonitis is treated with steroids, whereas SARS-CoV2 is not.

A second issue is related to the apparent similarity between the appearance of cytokine release syndrome in some patients receiving ICIs and a similar "cytokine storm" seen in patients with advanced COVID-19. ${ }^{30}$ Both appear to be the result of the release of highly active cytokines such as IL-6 as well as T cell activation, and there is evidence that immune hyperactivation mechanisms may be in part responsible for COVID-19 severity. The potential for an exacerbation of COVID-19-related cytokine storm in the event of a cytokine release syndrome reaction to ICI (or CAR-T cell therapy) is at least of 
hypothetical concern. Fortunately, cytokine release syndrome is a rare morbidity of ICI, and cytokine storm a late manifestation of COVID-19. Therefore, the risk of the two overlapping would seem to be small. While the potential for increased morbidity of ICI in the setting of COVID-19 should be carefully evaluated, concern should not prevent the administration of these potentially highly active agents in those cancer patients who can derive a significant benefit. ${ }^{30}$ Clinical judgement and a risk-benefit assessment must be individualized for the patient.

\section{COVID-19 and Radiotherapy}

Radiation therapy typically requires daily visits to the treatment center for multiple weeks, thereby putting patients and staff at risk of COVID transmission. Strategies are considered to deliver fractionated radiation in fewer doses. The goal of alternative radiation treatment schemes is preservation of clinical efficacy while reducing the number of daily treatments by increasing the dose per fraction. Guidance for alternative approaches requires adherence to acceptable radiobiological parameters and clinical tolerability.

Patients treated with thoracic radiotherapy may develop pneumonitis that could be confused with severe acute respiratory syndrome caused by this novel coronavirus. CT scan and clinical course should help differentiate these conditions. The onset of radiotherapy-induced pneumonitis is much slower than COVID-19 pneumonia, which typically develops rapidly over days; And chest CT findings observed with COVID-19 pneumonia may be diagnostic: ground-glass opacification, consolidation, bilateral involvement, and peripheral and diffuse distribution. ${ }^{31}$

\section{Disease-Specific Considerations}

Lung cancer presents a particular challenge in the setting of COVID-19; lung cancer is the most common cancer type in the USA, and therefore the risk of contracting SARS-CoV-2 in the setting of lung cancer is significant. Whether the modality employed is surgery, chemotherapy, radiation, or immunotherapy, the potential for exacerbation of lung inflammation and its impact on lung function is real. ${ }^{28,32}$ Absent COVID-19, patients with lung cancer are already at risk of pneumonitis, postprocedure infection, and loss of lung capacity based on comorbidities, smoking, and as a result of the modalities employed to treat their tumors. Principles of maintaining lung function through oncologically sound resections that still preserve lung volume such as video-assisted thoracoscopic surgery (VATS) take on an even greater degree of importance.

Methodologies to deliver fractionated radiation to a confined area of the chest, such as intensity-modulated radiation therapy (IMRT) and proton beam therapy, are important to consider to reduce injury to normal lung tissue. For lung cancer patients with known SARS-CoV-2 or active COVID-19, consideration of delay in radiotherapy for a few weeks until symptoms resolve and inflammation subsides is important. While risks and benefits of immunotherapy have already been discussed, it is important to note that these strategies play a particularly important role in the modern multimodality management of lung cancer. Risk-benefit assessment is therefore of paramount importance in this patient population.

Management of head and neck cancer patients is more complicated during the COVID pandemic. ${ }^{33}$ Those treating these patients are at increased risk of transmission through aerosolized particles, mucus, and blood. Operations involving any portion of the upper aerodigestive tract pose an increased risk of COVID transmission to those in the operating room. A high rate of transmission to otolaryngologists was reported in China, Italy, and Iran, with reports of morbidity and death. ${ }^{34}$ Planned radiation therapy should be delivered without delay when possible. Each month of delay in treatment is associated with a $16 \%$ increase in mortality. ${ }^{35}$

For patients with other advanced-stage malignancies, questions often arise as to whether cancer therapy can be safely postponed. This decision must be based on a number of factors, including cancer type, stage, and alternative to standard regimens that have some evidence of efficacy. While these decisions are individualized, some general principles apply. If treatment can be delayed based on indolent histology or early stage, then that is a reasonable option. For some regimens, a switch from IV to an oral route alternative may be possible, or it may be feasible to administer IV medications at home. Alternatively, IV chemotherapy dosing schedules that are every 3 weeks rather than weekly should be considered when appropriate. It may be reasonable to shorten adjuvant therapy based on reasonable extrapolation of clinical trial data, or even completely eliminate adjuvant therapy if the survival benefits are very small. Careful risk-benefit assessments must be made.

In many patients, delay or at-home options will not be feasible. For such cases, patients should be carefully monitored, protected from exposure if they are not SARSCoV-2 positive, and appropriately isolated from other patients if they are. For patients with active COVID-19 requiring active therapy for a cancer diagnosis, agents that can exacerbate the pulmonary sequelae of COVID-19 should be avoided until symptoms of the infection have resolved. Agents that can result in immunosuppression should be avoided when possible. 


\section{ETHICAL CONSIDERATIONS OF GUIDELINES}

The current COVID-19 pandemic has led several professional societies to provide guidance for cancer care for patients in anticipation of a public health crisis. ${ }^{13,36}$ These guidelines utilize limited available data and common-sense approaches to reduce the risks to patients and healthcare workers while optimizing healthcare resources in anticipation of the crisis. In fact, the three ethical duties of healthcare leaders include the duty to plan, the duty to safeguard, and the duty to guide. ${ }^{37}$ The guidelines are particularly relevant in decisions of triage, which are ethically the most challenging, when we switch from patient care ethics to public health ethics. However, guidelines to cope with an emerging pandemic, when based on limited evidence such as case reports and poorly controlled studies, are fraught with ethical concerns, some of which are discussed below (Fig. 2). ${ }^{38}$

\section{Ethical Concerns of Guidelines for Individual Patient Care}

The greatest risk of guidelines is that they may be flawed or wrong for the individual patient. In the instance of COVID-19, this risk is particularly concerning. The perceived risk of harm to cancer patients with COVID-19 undergoing surgery comes from small case series, which are relatively poorly designed studies. ${ }^{39}$ Publications in high-impact journals trying to rapidly disseminate information can falsely elevate the perception of higher quality of evidence. Guidelines are rarely updated expeditiously, which is necessary for a rapidly evolving disease. When guidelines are inflexible ("should," as opposed to "may"), they can compromise individual patient preferences, affect policy including insurance coverage, lead to redistribution of resources, or be misused by specific advocacy groups.

While most members of guideline committees are experienced clinicians, their local experiences will temper their recommendations. Hence, an expert from New York (1354 cases/100,000 people) might have a completely separate assessment of the public health crisis than an expert in Texas (76 cases/100,000 people). Most importantly, guidelines are designed to account for perspectives other than those of the patient, including those of the healthcare system, the lawmakers, national societies, and business leaders. This can obviously disadvantage the individual patient who is advocating for his or her own health. Additionally, guidelines can encourage and embed institutionally, ineffective, harmful, or wasteful practices. Examples of such interventions include prophylaxis with hydroxychloroquine and routine antibody testing without validated tests.

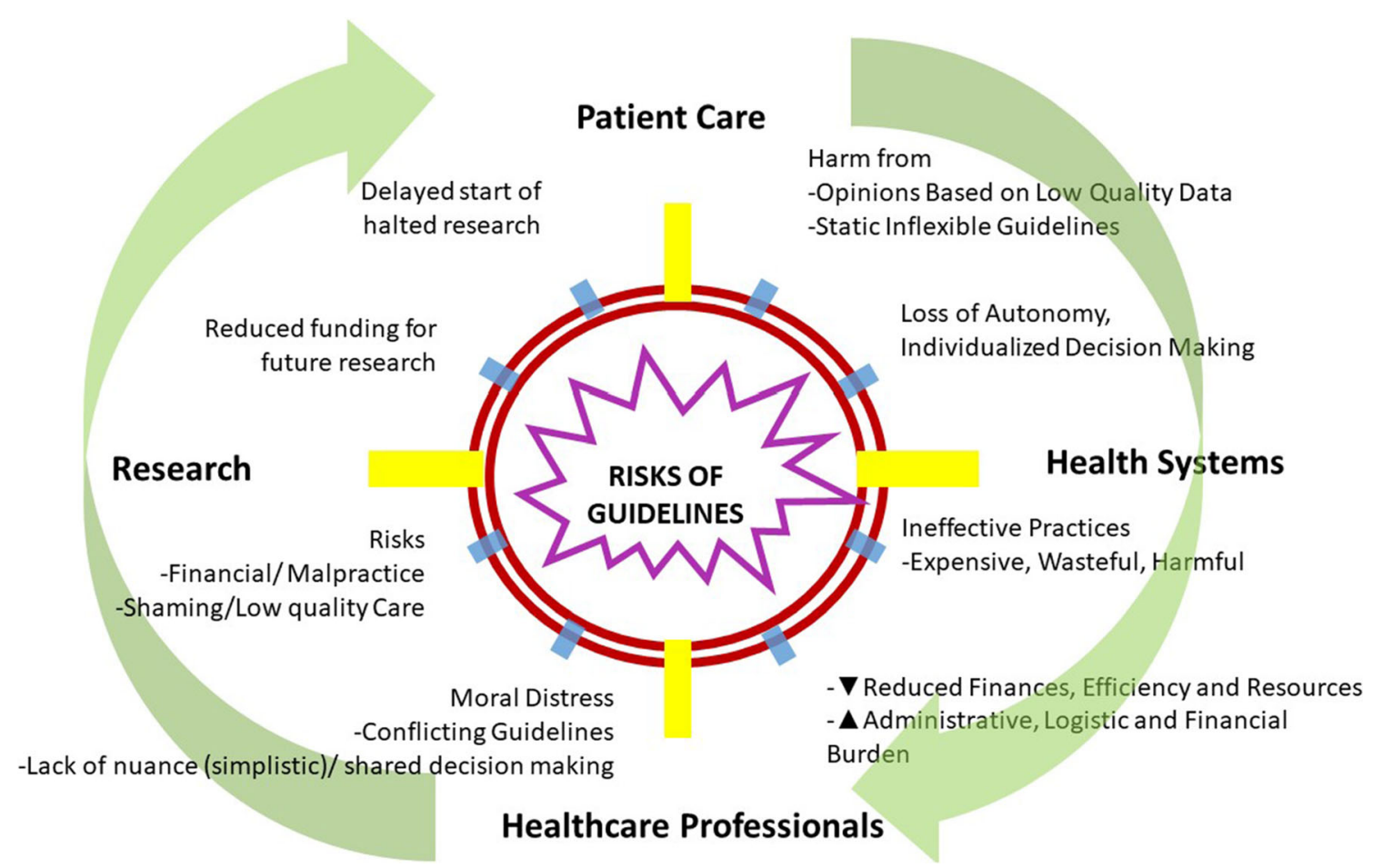

FIG. 2 Ethical risks of guidelines developed and implemented during uncertain times 
Ethical Concerns of Guidelines for Healthcare Professionals

Guidelines can be extremely helpful in reducing variance and providing guidance in times of crisis but, if flawed or based on poor evidence, can lead to wasteful and harmful practices. Numerous conflicting guidelines can be frustrating for healthcare personnel, especially when they interfere with an autonomous doctor-patient relationship. Additionally, guidelines tend to be simplistic (binary yes/ no, Likert scale-based triage systems) and may not allow for the nuances of individualized care required for patients. These generally do not allow for iterative sequencing of care and shared decision-making with patients and their families, both of which form the backbone of surgical oncology.

This can lead to significant moral distress for physicians, specifically cancer surgeons. Postponing time-sensitive operations or diverting patients to less effective pathways and reducing accrual to potentially life-saving clinical trials are specific circumstances that can add to the distress of the healthcare team. Such guidelines can place clinicians in a position of being judged unfairly by their peers or the health system and pose theoretical malpractice risks when clinicians do not adhere to them. The financial ramifications of guidelines that create delays to surgery whenever feasible cannot be understated, especially for solo practitioners and small practices, who may lose their livelihood based on triaging patients and diverting them towards alternative treatments. Finally, the quality of cancerspecific outcomes can be adversely impacted by poorly designed or overly generalized guidelines.

\section{Ethical Concerns of Guidelines for Healthcare Systems}

Healthcare systems are affected by the beneficial and harmful effects of guidelines in a public health crisis. Triage and diversion of cancer care away from surgery can have a profound impact. Cancer surgery remains one of the pillars of a cancer center. Guidelines that impose reduced utilization of cancer surgery can dramatically impact the goals of the institution through creation of new financial, logistic, and administrative burdens. Furthermore, longterm acceptance of altered practice patterns through habit rather than evidence even after the crisis has passed could well prolong patterns of care that are in neither the patient's nor the institution's best interests.

\section{Ethical Concerns of Policies for Scientific Investigation and Research}

Policies that create barriers to cancer research during the COVID-19 epidemic may disadvantage patients and investigators even after the crisis has passed. Patient and staff safety are of paramount importance. However, through the turmoil and human suffering created by the COVID-19 pandemic, it is easy to lose sight of the fact that 10 million people will die from cancer this year alone. Moreover, successes in cancer research can have a dramatic impact on millions of people. Patients may derive benefit from participation in even early-phase clinical trials. Continued collection of biospecimens-in particular during the pandemic-is the backbone of future breakthroughs in discovery of targets and biomarkers.

Clinical guidelines and institutional policies have necessarily been drafted quickly and with limited data to improve patient and public health outcomes and reduce harm to patients, healthcare providers, and society. It is important to recognize the limitations of these guidelines, continually reassess their validity, and modify or discard those that do not demonstrate their value with scientific scrutiny.

\section{ADVOCACY FOR CANCER PATIENTS IN TIME OF COVID-19 PANDEMIC}

Surgical oncologists find themselves as primary advocates for patients who are in need of cancer operations during this pandemic. Physician and hospital leaders as well as policymakers must understand the important needs of cancer patients, whose diagnosis, staging, and treatment depend upon the timely availability of surgical care to maximize a successful outcome. Otherwise, they become a statistic of "preventable death," just the same as an individual facing severe attack from the COVID-19 virus infection. For most types of cancer, there is solid evidence of a window of time of approximately 4-8 weeks from the time of diagnosis to definitive surgical treatment. Published reports provide evidence that survival rates are diminished for patients whose definitive operations are delayed beyond this window. ${ }^{40-42}$ Patient safety during this pandemic is clearly of paramount importance; but safety is a two-edged sword. Safety derived from pursuit of institutional goals of social distancing, conservation of hospital resources, and preservation of PPEs are undoubtedly important. However, the safety of cancer patients is of particular concern when treatment is put on hold in face of a progressive disease process; delays in standard treatment may compromise their chances for survival. Identification of a medical middle ground that best addresses the multiple needs is appropriate.

Cancer surgery generally does not fall into the category of emergency surgery (unless there is a life-threatening condition), nor is it in the category of elective surgery (similar to a hernia repair). Rather, most operations in 
cancer patients may be categorized as time sensitive and essential. Nevertheless, policies issued by many state departments of public health or implemented by hospitals require postponing elective surgery during the COVID-19 pandemic but recognizing the need for emergency surgery to proceed. Hospital committees, licensing boards, and policymakers have not uniformly integrated the important circumstance of cancer patients with surgically treatable disease and the need to give priority access to these patients.

Patients who complete neoadjuvant therapy also find themselves in need of an operation that is time sensitive. The appropriate window for operation following neoadjuvant therapy in many cases is well defined and relatively small (e.g., neoadjuvant chemoradiation therapy for rectal cancer). Thus, gaps in time between the completion of appropriate course of neoadjuvant therapy and operation may impact survival statistics and may impact operative morbidity and mortality. Nonetheless, there are other situations in which the window of time for operation is much larger following neoadjuvant therapy (e.g., hormone-sensitive breast cancer or low-grade prostate cancer).

Reliance on multidisciplinary teams to provide recommendations on delays carries many advantages, as outlined above. Another approach involves the development and use of a scoring system for triage. As an example, Emory University Healthcare created a surgical prioritization tool based on patient age, cancer type (if present), and the time after which delay will either impact patient survival or result in an irreversible compromise in outcome that impacts patient function. The scoring tool is built into a database that allows tracking, storing, filtering, and sorting of the pending cases to help construct operating room schedules at the various hospital sites based on medical prioritization and is modified by any local resource constraints (OR availability, ICU availability, bed availability, blood availability, etc.). This technology has limited the bias inherent to these complex decisions, while creating an efficient method for scheduling and prioritizing these cases.

\section{CONCLUSIONS}

SARS-CoV-2 infection and the COVID-19 pandemic have created unprecedented challenges for delivery of cancer care. The human toll caused by the COVID-19 pandemic is overwhelming and has claimed almost 300,000 lives worldwide at the time of this writing. However, we must also remember that 10 million people worldwide will die of cancer this year alone. It is incumbent on individual providers and the healthcare system to deliver optimal cancer treatment while working within government and institutional policies appropriately designed to reduce infections, ensure sufficient resources, and provide safety for patients and healthcare staff. This involves supporting a healthy workforce, leveraging multidisciplinary teams to triage effectively, innovating new solutions for patient care and communication, and advocating for our cancer patients as well as cancer operations as appropriate. Policies and guidelines developed in response to the pandemic should be continually reassessed to ensure they remain loyal to their objectives under careful scrutiny. The pandemic has been a catalyzing force which will result in tremendous and lasting change in healthcare delivery; the opportunity must be seized to address many of the serious problems in healthcare that may be less intractable than we believed prior to COVID-19.

DISCLOSURES The authors have no conflicts of interest to disclose.

\section{REFERENCES}

1. National Institutes of Health NCI, Surveillance, Epidemiology, and End Results Program. Cancer stat facts: common cancer sites 2020. Available from: https://seer.cancer.gov/statfacts/html/com mon.html.

2. Carolan A. Conversation with the cancer letter. What to expect: oncology's response to coronavirus in Italy. Cancer Lett. 1919 March 11. 2020.

3. European Society of Medical Oncology. Cancer patient management during the COVID-19 pandemic 2020. Available from: https://www.esmo.org/guidelines/cancer-patient-management-du ring-the-covid-19-pandemic.

4. Lauer SA, Grantz KH, Bi Q, Jones FK, Zheng Q, Meredith HR, et al. The incubation period of coronavirus disease 2019 (COVID-19) from publicly reported confirmed cases: estimation and application. Ann Int. Med. 2020;172:577-582.

5. Cook TM. Personal protective equipment during the COVID-19 pandemic-a narrative review. Anaesthesia. 2020.

6. Hsu T. Where thousands of masks a day are decontaminated to battle the virus. The New York Times. 2020 April 11.

7. Tate C. Hilton, Marriott donate free hotel rooms for medical workers responding to coronavirus crisis. USA Today. 2020.

8. American College of Surgeons. COVID-19: elective case triage guidelines for surgical care 2020. Available from: https://www. facs.org/covid-19/clinical-guidance/elective-case.

9. American Society of Anesthesiologists. COVID-19 FAQs 2020. Available from: https://www.asahq.org/about-asa/governance-an d-committees/asa-committees/committee-on-occupational-healt $\mathrm{h} /$ coronavirus/clinical-faqs\#gen.

10. Lai J, Ma S, Wang Y, Cai Z, Hu J, Wei N, et al. Factors associated with mental health outcomes among health care workers exposed to coronavirus disease 2019. JAMA Netw Open. 2020;3(3):e203976.

11. Ueda M, Martins R, Hendrie PC, McDonnell T, Crews JR, Wong TL, et al. Managing cancer care during the COVID-19 pandemic: agility and collaboration toward a common goal. J Natl Compr Cancer Netw. 2020;18(4):1-4.

12. Akladios C, Azais H, Ballester M, Bendifallah S, Bolze PA, Bourdel N, et al. Recommendations for the surgical management of gynecological cancers during the COVID-19 pandemicFRANCOGYN group for the CNGOF. J Gynecol Obstet Hum Reprod. 2020. https://doi.org/10.1016/j.jogoh.2020.101729. 
13. Bartlett DL, Howe JR, Chang G, Crago A, Hogg M, Karakousis $\mathrm{G}$, et al. Management of cancer surgery cases during the COVID19 pandemic: considerations. Ann Surg Oncol. 2020;27:1717-20.

14. American College of Surgeons. COVID-19 guidelines for triage of cancer surgery patients 2020. Available from: https://www.fac s.org/covid-19/clinical-guidance/elective-case/cancer-surgery.

15. Thoracic Surgery Outcomes Research Network Inc. COVID-19 guidance for triage of operations for thoracic malignancies: a consensus statement from thoracic surgery outcomes research network. Ann Thorac Surg. 2020. https://doi.org/10.1016/j.athor acsur.2020.03.005.

16. Topf MC, Shenson JA, Holsinger FC, et al. Framework for prioritizing head and neck surgery during the COVID-19 pandemic. Head Neck. 2020;42(6):1159-1167. https://doi.org/10.1002/hed. 26184.

17. Yang S, Cao P, Du P, Wu Z, Zhuang Z, Yang L, et al. Early estimation of the case fatality rate of COVID-19 in mainland China: a data-driven analysis. Ann Transl Med. 2020;8(4):128.

18. Yu J, Ouyang W, Chua MLK, Xie C. SARS-CoV-2 Transmission in patients with cancer at a tertiary care hospital in Wuhan, China. JAMA Oncol. 2020.

19. Zhang L, Zhu F, Xie L, Wang C, Wang J, Chen R, et al. Clinical characteristics of COVID-19-infected cancer patients: a retrospective case study in three hospitals within Wuhan, China. Ann Oncol. 2020. https://doi.org/10.1016/j.annonc.2020.03.296.

20. Mazzaferro V, Danelli P, Torzilli G, Busset MDD, Virdis M, Sposito C. A combined approach to priorities of surgical oncology during the COVID-19 epidemic. Ann Surg. 2020.

21. Kane CK, Gillis K. The use of telemedicine by physicians: still the exception rather than the rule. Health Aff (Millwood). 2018;37(12):1923-30.

22. University of New Mexico Health Science Center ECHO Institute. Project Echo 2020. Available from: https://echo.unm.edu/.

23. Asiri A, AlBishi S, AlMadani W, ElMetwally A, Househ M. The use of telemedicine in surgical care: a systematic review. Acta Inform Med. 2018;26(3):201-6.

24. U.S. Food and Drug Administration. FDA Guidance on Conduct of Clinical Trials of Medical Products during COVID-19 Public Health Emergency. 2020. https://www.fda.gov/media/136238/do wnload.

25. Center for Medicare and Medicaid Services. Medicare telemedicine health care provider fact sheet 2020. Available from: $h$ ttps://www.cms.gov/newsroom/fact-sheets/medicare-telemedicin e-health-care-provider-fact-sheet.

26. Federation of State Medical Boards. States modifying requirements for telehealth in response to COVID-19 2020. Available from: https://www.fsmb.org/siteassets/advocacy/pdf/states-waivi ng-licensure-requirements-for-telehealth-in-response-to-covid-19 .pdf.

27. Shankar A, Saini D, Roy S, Mosavi Jarrahi A, Chakraborty A, Bharti SJ, et al. Cancer care delivery challenges amidst coronavirus disease-19 (COVID-19) outbreak: specific precautions for cancer patients and cancer care providers to prevent spread. Asian Pac J Cancer Prev. 2020;21(3):569-73.

28. Sidaway P. COVID-19 and cancer: what we know so far. Nat Rev Clin Oncol. 2020;17:336.
29. Xia Y, Jin R, Zhao J, Li W, Shen H. Risk of COVID-19 for patients with cancer. Lancet Oncol. 2020;21(4):e180.

30. Bersanelli M. Controversies about COVID-19 and anticancer treatment with immune checkpoint inhibitors. Immunotherapy. 2020;12:269-73

31. Shi H, Han X, Jiang N, Cao Y, Alwalid O, Gu J, et al. Radiological findings from 81 patients with COVID-19 pneumonia in Wuhan, China: a descriptive study. Lancet Infect Dis. 2020;20(4):425-34.

32. Tian $\mathrm{S}, \mathrm{Hu} \mathrm{W}$, Niu L, Liu $\mathrm{H}, \mathrm{Xu} \mathrm{H}$, Xiao SY. Pulmonary pathology of early-phase 2019 novel coronavirus (COVID-19) pneumonia in two patients with lung cancer. $J$ Thorac Oncol. 2020;15(5):700-4.

33. Yuen E, Fote G, Horwich P, Nguyen SA, Patel R, Davies J, et al. Head and neck cancer care in the COVID-19 pandemic: a brief update. Oral Oncol. 2020;105:104738.

34. American Academy of Otolaryngology-Head and Neck Surgery. AAO-HNS Position statement: otolaryngologists and the COVID-19 pandemic 2020; March 23, 2020. Available from: h ttps://www.entnet.org/content/aao-hns-position-statement-otolary ngologists-and-covid-19-pandemic.

35. Chen Z, King W, Pearcey R, Kerba M, Mackillop WJ. The relationship between waiting time for radiotherapy and clinical outcomes: a systematic review of the literature. Radiother Oncol. 2008;87(1):3-16

36. Marron JM, Joffe S, Jagsi R, Spence RA, Hlubocky FJ. Ethics and resource scarcity: ASCO recommendations for the oncology community during the COVID-19 pandemic. J Clin Oncol. 2020. https://doi.org/10.1200/JCO.20.00960.

37. Berlinger N, Wynia M, Powel T, Hester M, Milliken A, Fabi R, et al. Ethical framework for health care institutions responding to novel coronavirus SARS-CoV-2 (COVID-19) guidelines for institutional ethics services responding to COVID-19. The Hastings Center [Internet]. 2020. pp. 1-12. Available from: $h$ ttps://www.thehastingscenter.org/ethicalframeworkcovid19/.

38. Woolf SH, Grol R, Hutchinson A, Eccles M, Grimshaw J. Clinical guidelines: potential benefits, limitations, and harms of clinical guidelines. BMJ. 1999;318(7182):527-30.

39. Liang W, Guan W, Chen R, Wang W, Li J, Xu K, et al. Cancer patients in SARS-CoV-2 infection: a nationwide analysis in China. Lancet Oncol. 2020;21(3):335-7.

40. Bleicher RJ. Timing and delays in breast cancer evaluation and treatment. Ann Surg Oncol. 2018;25(10):2829-38.

41. Grass F, Behm KT, Duchalais E, Crippa J, Spears GM, Harmsen WS, et al. Impact of delay to surgery on survival in stage I-III colon cancer. Eur J Surg Oncol. 2020;46(3):455-61.

42. Kaltenmeier C, Shen C, Medich DS, Geller DA, Bartlett DL, Tsung A, et al. Time to surgery and colon cancer survival in the United States. Ann Surg. 2019. https://doi.org/10.1097/SLA. 0000000000003745 .

Publisher's Note Springer Nature remains neutral with regard to jurisdictional claims in published maps and institutional affiliations. 